\title{
3D micro-printed hybrid photonic structure for single-fiber Optical Tweezers
}

\author{
Innem V.A.K Reddy ${ }^{1,2}$, Andrea Bertoncini ${ }^{1}$, Carlo Liberale ${ }^{1,3}$ \\ 1. Biological and Environmental Science and Engineering Division, King Abdullah University of Science and Technology, Saudi Arabia \\ 2. Department of Electrical Engineering, University at Buffalo, NY USA \\ 3. Computer, Electrical and Mathematical Science and Engineering Division, King Abdullah University of Science and Technology, Saudi \\ Arabia
}

Optical tweezers (OT) are non-destructive, contactless tools that use light to trap and manipulate microscopic objects. They have applications in diverse fields, particularly in biomedicine and physics [1]. Due to their unique applications and the ability to analyze sub-micron biological systems, the Nobel prize for Physics in 2018 was awarded to Arthur Ashkin, the inventor of OT. Conventional single-beam OT setups require the focusing of a laser beam with a high-Numerical Aperture (NA) objective. However, these setups are bulky and restrict the trapping capabilities to table-top arrangements. The miniaturization of OT to be implemented with a single optical fiber is vital as it opens up new possibilities such as trapping in turbid media or in-vivo. Several groups demonstrated single-fiber OT but the proposed approaches use non-standard fibers and grant only limited design flexibility in controlling the optical trap geometry [2].

We present a novel 3D micro-printed hybrid photonic structure creating ultra-compact fiber tweezers with fully controllable optical parameters. The structure is made of stacked refractive, reflective, and waveguiding optical elements (see Fig. 1a) and is fabricated in a single step on the end-face of the fiber (Fig. 1b) with a commercial two-photon lithography system (Nanoscribe). The design of the different parts of the hybrid structure allows an easy and independent tuning of the trapping parameters as the NA and the working distance of the optical trap. In particular, a waveguide segment with photonic crystal fiber design [3] converts the Gaussian-like beam output from a standard single-mode fiber into an annular beam with $10.5 \mu \mathrm{m}$ radius. In the second segment, the annular beam's diameter is significantly enlarged (up to $113 \mu \mathrm{m}$, see Fig. 1c) by exploiting total internal reflection (TIR) within the structure. The parabolic profile of one of the two reflecting surfaces allows for the collimation of the beam. In the final segment, an axially symmetric prism-like structure deflects the annular beam by TIR to create a high-NA converging beam at the trapping point. We demonstrate the 3D trapping capabilities of the proposed fiber OT (Fig. 1d).

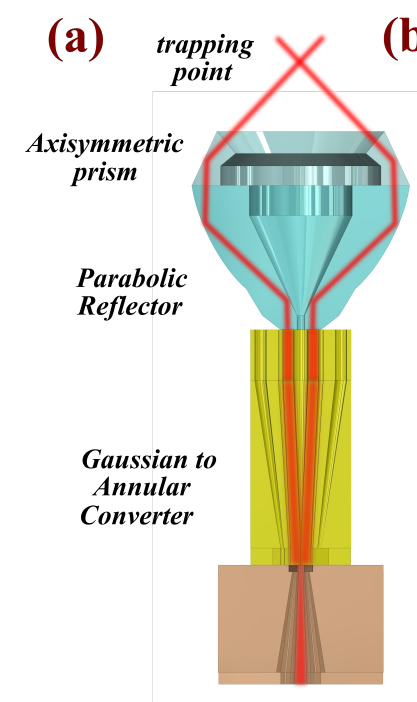

(b)

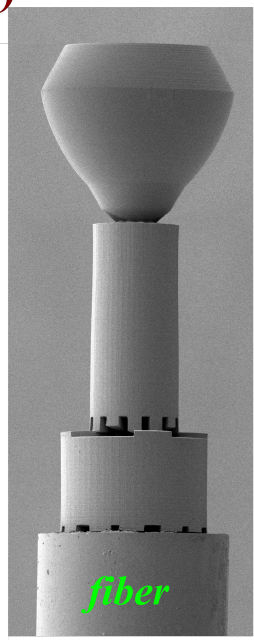

(c)

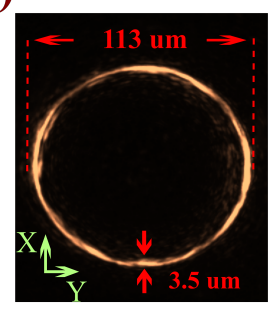

(d)

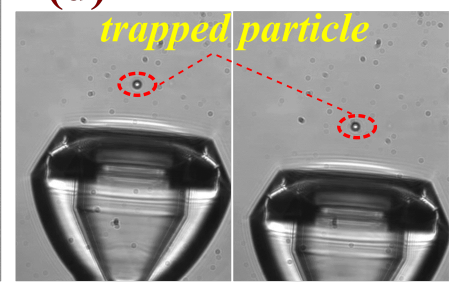

Figure 1: (a) Design of the fiber OT, (b) SEM image of the structure fabricated on the tip of the fiber, (c) Annular beam output from the parabolic reflector, (d) Optical trapping of a $5 \mu \mathrm{m}$ polystyrene bead.

\section{References}

[1] A. Ashkin, "History of optical trapping and manipulation of small-neutral particle, atoms, and molecules," Ieee Journal of Selected Topics in Quantum Electronics 6(6), 841-856, (2000).

[2] X.T. Zhao, N. Zhao, Y. Shi, H. B. Xin and B. J. Li, "Optical Fiber Tweezers: A Versatile Tool for Optical Trapping and Manipulation," Micromachines 11(2), (2020).

[3] A. Bertoncini, C. Liberale, "3D printed waveguides based on photonic crystal fiber designs for complex fiber-end photonic devices," Optica 7, 1487-1494 (2020). 